\title{
EL EFECTO DE LA IMPLEMENTACIÓN DE LA GUÍA INFORMATIVA DE ALTA EN EL CUMPLIMIENTO DE LAS INDICACIONES DE CUIDADOS PUERPERALES Y CUIDADOS DEL RECIÉN NACIDO DE LAS PUÉRPERAS MEDIATAS ATENDIDAS EN EL SERVICIO DE ADOLESCENCIA EN EL INSTITUTO NACIONAL MATERNO PERINATAL - 2012
}

\author{
Aydee Hinojosa Ruelas ${ }^{1}$, Ana Clarice Paiva Pacaya ${ }^{1}$, Celinda Paola Ruiz Aguilar ${ }^{1}$, \\ Jessica Mariela Salazar Torres ${ }^{1}$
}

\begin{abstract}
RESUMEN
Objetivos. Determinar el efecto de la implementación de la guía informativa sobre el cumplimiento de las indicaciones de cuidados puerperales y del recién nacido de las puérperas mediatas adolescentes atendidas en el servicio de adolescencia del Instituto Nacional Materno Perinatal-2012. Materiales y métodos. Estudio cuasi-experimental, prospectivo, comparativo, se contó con un grupo experimental y un grupo comparativo. Se evaluaron a 120 puérperas a razón de 60 puérperas adolescentes en cada grupo, divididas en dos grupos: Grupo A, pacientes a las que se aplicó una guía informativa y grupo B pacientes a las cuales no se les aplicó la guía informativa, todas atendidas en el INMP desde octubre 2011 a febrero 2012. Se estimó para las variables cualitativas las frecuencias absolutas y relativas. Para el caso de variables cuantitativas se estimaron las medidas de tendencia central como media, mediana, y medidas de dispersión como desviación estándar y rango. Para el análisis inferencial se utilizó la prueba t de student para muestras independientes y prueba de independencia chi cuadrado. Resultados. Mayoría de pacientes procedían de los distritos de SJL 31,7\%, SJM 11,7\% y Villa el Salvador 10\%; edad promedio 16,72 $\pm 1,3$ años; $80 \%$ tuvieron educación secundaria; $70 \%$ fueron convivientes. El $63,3 \%$ de los partos fue vaginal y el $36,7 \%$ por cesárea; estancia hospitalaria promedio fue 3,43 $\pm 1,5$ días. En el grupo que se aplicó la guía de orientación se alcanzó el 63,3\% de cumplimiento calificado como "Muy bueno", comparado con el 6,7\% del grupo que no se aplicó la guía de orientación. El nivel de cumplimiento sobre los cuidados del recién nacido se alcanzó el $66,7 \%$ de cumplimiento calificado como "Muy bueno" comparado con el 16,7\% del grupo control, observándose que existe un efecto positivo de la implementación de la guía de informativa de alta en el cumplimiento de las indicaciones y cuidados puerperales, encontrándose relación entre ambas variables $(p<0,001)$. Asimismo, existe un efecto positivo de la implementación de la guía de informativa de alta en el cumplimiento de las indicaciones y cuidados del recién nacido, relacionándose ambas variables $(p<0,001)$. Conclusiones. El nivel de cumplimiento de la guía de orientación sobre el cuidado puerperal y del recién nacido alcanzó efecto positivo relevante.
\end{abstract}

Palabras Claves: Cuidados; Puerperio; Recién nacido; Guía (fuente: DeCS BIREME).

\section{THE EFFECT OF THE IMPLEMENTATION OF THE HIGH INFORMATION GUIDE IN COMPLIANCE PUERPERAL CARE INDICATIONS AND NEWBORN CARE MEDIATE POSTPARTUM WOMEN SERVED IN THE SERVICE OF ADOLESCENT MOTHER IN THE MATERNAL PERINATAL NATIONAL INSTITUTE - 2012}

\begin{abstract}
Objectives. To determine the effect of the implementation of the information brochure on compliance with the directions of postnatal care and newborn mediate the postpartum adolescents seen in adolescence Service National Perinatal Maternal Institute, 2012. Materials and methods. A quasi-experimental, prospective, counted with an experimental group and a comparison group. 120 postpartum women were evaluated at 60 postpartum adolescents in each group, divided into two groups: Group A patients was applied to an information and group B patients which were not subject to the information package, all served in the INMP from October 2011 to February 2012. Was estimated for qualitative variables, absolute and relative frequencies. In the case of quantitative variables were estimated as measures of central tendency mean, median, and measures of dispersion such as standard deviation and range. For the inferential analysis used the Student t test for independent samples and chi-square test of independence. Results. Most patients came from the districts of SJL $31,7 \%, 11,7 \%$ and SJM Villa el Salvador $10 \%$, mean age $16,72 \pm 1.3$ years, $80 \%$ had secondary education, $70 \%$ were cohabiting. The $63,3 \%$ of deliveries were vaginal and $36.7 \%$ by caesarean average hospital stay was $3,43 \pm 1,5$ days. In the group that was applied orientation guide reached $63,3 \%$ compliance rated "Very Good", compared with $6,7 \%$ in the group that did not undergo orientation guide. The level of compliance on newborn care was achieved $66,7 \%$ compliance rated "Very Good" compared with $16,7 \%$ in the control group, showing that there is a positive effect of the implementation of the informative guide High in compliance with the indications and postnatal care, finding relationship between the two variables $(p<0,001)$. There is also a positive effect of the implementation of high-informative guide in meeting the indications and newborn care, relating both variables $(p<0,001)$. Conclusions. The level of compliance orientation guide postpartum care and newborn reached significant positive effect.
\end{abstract}

Key words: Care; Postpartum; Newborn; Guide (source: MeSH NLM).

Licenciada en Enfermería. Servicio de Obstetricia A - Adolescencia. Instituto Nacional Materno Perinatal. Lima-Perú.

Recibido: 10-10-12 Aprobado: 22-04-13 


\section{INTRODUCCIÓN}

La Organización Mundial de la Salud (2011) considera la adolescencia como la etapa que transcurre entre los 10 y 19 años, que es un periodo de transición entre la infancia y la edad adulta. Es un período en el que se presentan características peculiares como experimentar e incorporar cambios interiores, es en esta etapa donde se da la búsqueda de la identidad; psicológica y sexual; cambios exteriores y corporales (OMS 2011, Valverde 2008).

El Ministerio de Protección Social de la República de Colombia en el 2010 contempló en su Plan Obligatorio de Salud (POS) la elaboración de guías de atención integral para la prevención y detección temprana de las alteraciones del embarazo y complicaciones del parto y puerperio (Martínez, 2009).

Así mismo en Bolivia la implementación de protocolos y guías en la prestación de servicios de atención maternaneonatal, ayudaron a incrementar las coberturas de atención en salud materna y reducir enfermedades y muertes de madres y niños si son atendidos oportunamente, con calidad, calidez, respeto y valoración a los conocimientos locales en salud (Zalles, 2006).

El gobierno de El Salvador presento las "Guías de Atención de los Principales Problemas de Salud de Adolescente". Dichas Guías tenían la finalidad de orientar a los proveedores de salud para el abordaje de atención de adolescentes, permitiendo la detección oportuna de signos y síntomas y el manejo adecuado para el restablecimiento de la salud. Además contribuye a fortalecer los procesos de atención en los ámbitos: Familiar, Educativo, Laboral y Establecimiento de Salud (Betancourt, 2003).

En el servicio de adolescencia del Instituto Nacional Materno Perinatal no existe un plan de alta que incluya la aplicación de una guía de alta que sea comprendida con calidad y acorde a la característica principal del Servicio, por eso su implementación es oportuna y necesaria. En este sentido el objetivo de la investigación fue determinar el efecto de la implementación de la guía informativa de alta en el cumplimiento de las indicaciones de cuidados puerperales y cuidados del recién nacido de las puérperas mediatas adolescentes atendidas en el Servicio de Adolescencia del Instituto Nacional Materno Perinatal- 2012.

\section{MATERIALES Y MÉTODOS}

Diseño de Estudio. Estudio cuasi-experimental con post evaluación, para lo cual se contó con un grupo experimental y un grupo comparativo.

Muestra. La muestra fue no aleatoria y el estudio tuvo una duración de un mes.
Población de estudio. Puérperas mediatas dolescentes atendidas en el Servicio de Adolescencia del Instituto Nacional Materno Perinatal- 2012.

Tamaño de la muestra. 120 puérperas adolescentes. A razón de 60 puérperas adolescentes en cada grupo. Divididas en dos grupos A y B. Para el estudio del Grupo A se aplicó la guía informativa de alta, el cual fue considerado grupo experimental y para el grupo B fue considerado como grupo comparativo.

Tipo de muestreo. El tipo de muestreo fue probabilístico y la técnica a utilizar fue por conveniencia.

\section{Criterios de Inclusión}

- Puérpera adolescente.

- Primípara.

- Controlada en el INMP (mínimo 4 controles).

- Puérpera que no haya presentado complicaciones en el postparto mediato.

- Puérpera y recién nacido en condiciones de alta.

- Participación voluntaria en el estudio.

\section{Criterios de Exclusión}

- Puérpera mayor de 20 años.

- Multigesta.

- No Controlada en el INMP.

- Puérpera que haya presentado complicaciones en el postparto mediato.

- Puérpera que no desea participar voluntaria en el estudio.

\section{Técnica de recolección de datos}

Se utilizó la técnica de la entrevista para recoger información de fuente primaria. En el Servicio de adolescencia contamos con 47 camas, divididas en dos lados. El lado A corresponde a puerperio normal con 16 camas y gestantes de alto riesgo con 7 camas y el lado $B$ corresponde a postoperadas con 24 camas.

\section{Procesamiento y análisis de datos}

\section{Plan de procesamiento}

Para determinar el "nivel de cumplimiento" de las indicaciones de cuidados puerperales entregados en el alta, se realizó mediante la escala de likert modificada (si la distribución del puntaje total tiene distribución normal, sino se utilizó la escala no paramétrica de los percentiles 50 y 75 del puntaje máximo).

Para determinar el "nivel de cumplimiento" de las indicaciones de cuidados del recién nacido entregados en el alta, se realizó mediante la escala de Likert modificada (si la distribución del puntaje total tiene distribución normal, sino se utilizó la escala no paramétrica de los percentiles 50 y 75 del puntaje máximo). El puntaje máximo para la variable cumplimiento de las indicaciones de cuidados 
Tabla 1. Distrito de procedencia de las puérperas mediatas atendidas en el servicio de adolescencia del INMP- 2012.

\begin{tabular}{lcc}
\hline Distrito de procedencia. & N & $\%$ \\
\hline San Juan de Lurigancho & 19 & 31,7 \\
\hline San Juan de Miraflores & 7 & 11,7 \\
\hline Villa el Salvador & 6 & 10,0 \\
\hline La Victoria & 5 & 8,3 \\
\hline El Agustino & 4 & 6,7 \\
\hline Lima & 4 & 6,7 \\
Callao & 3 & 5,0 \\
\hline Comas & 2 & 3,3 \\
\hline Independencia & 2 & 3.3 \\
\hline Cercado & 1 & 1,7 \\
\hline Huaycan & 1 & 1,7 \\
\hline Los Olivos & 1 & 1,7 \\
Paramonga & 1 & 1,7 \\
\hline San Luis & 1 & 1,7 \\
\hline San Martin de Porres & 1 & 1,7 \\
\hline San Miguel & 1 & 1,7 \\
\hline Santa Anita & 1 & 1,7 \\
\hline
\end{tabular}

Fuente: Instrumento 2 de la Guía Informativa de Alta.

puerperales fue 25; y para la variable cumplimiento de las indicaciones del recién nacido fue 15 .

\section{Análisis de datos}

Los datos se registraron en una base elaborada en la hoja de cálculo del programa IBM Statistics SPSS 19 tomando en cuenta todas las variables e indicadores, realizando el análisis descriptivo y analítico con el mismo paquete estadístico.

\section{Aspectos Éticos}

Al ser un estudio prospectivo, fue importante contar con la aceptación de la paciente, para responder a las preguntas y

Tabla 2. Características Socio-demográficas de las puérperas mediatas atendidas en el servicio de adolescencia del INMP- 2012.

\begin{tabular}{lcc}
\hline Características & Promedio \pm DS (Mín. - Máx.) \\
Socio-demográficas & $16,72 \pm 1,318(13-19)$ \\
\hline Edad. & $3,43 \pm 1,5(1-7)$ \\
\hline Estancia Hospitalaria. & $\mathbf{N}$ & $\%$ \\
\hline Nivel educativo. & 7 & 11,67 \\
\hline Primaria & 48 & 80,00 \\
Secundaria & 0 & 0,00 \\
Superior Técnico & 1 & 1,67 \\
Superior Universitario & 4 & 6,67 \\
Sin Instrucción & & \\
Estado civil. & 17 & 28,33 \\
Soltera & 1 & 1,67 \\
Casada & 42 & 70,00 \\
\hline Conviviente & 60 & 100,00 \\
\hline Total & & \\
\hline
\end{tabular}

Fuente: Instrumento 2 de la Guía Informativa de Alta.

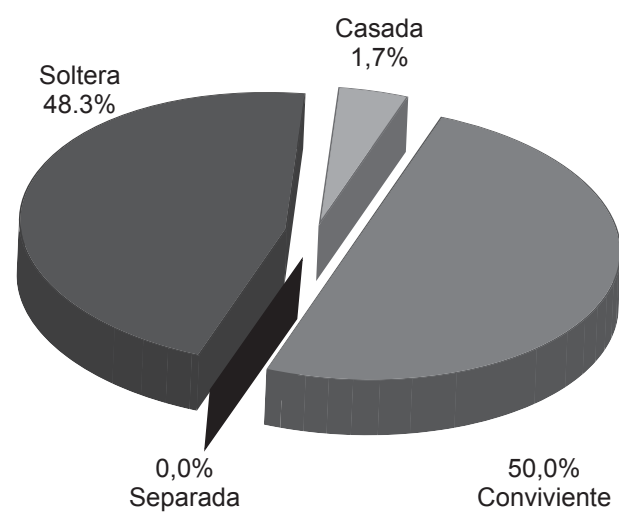

Figura 1. Estado Civil de las puérperas mediatas atendidas en el servicio de adolescencia del INMP- 2012.

Fuente: Instrumento 2 de la Guía Informativa de Alta.

su receptividad a la orientación. Se garantizó la confiabilidad de su respuesta y reserva de su opinión, se hizo extensivo el uso del consentimiento informado previo a la aplicación del instrumento del presente trabajo. Este documento nos garantizó la aceptación de la usuaria, la cual estuvo informada de los objetivos del proyecto. Se mantuvo la confidencialidad de la usuaria, ya que los cuestionarios fueron de forma anónima. La identidad de los informantes no fue revelada a fuentes externas por tener fines académicos.

\section{RESULTADOS}

El perfil socio demográfico que describe a las 60 pacientes evaluadas son procedentes principalmente de los distritos de San Juan de Lurigancho 31,7\%, San Juan de Miraflores $11,7 \%$ y Villa el Salvador $10 \%$; cuya edad promedio fue

Tabla 3. Comparativo de Características Sociodemográficas las de las puérperas mediatas atendidas en el servicio de adolescencia del INMP- 2012.

\begin{tabular}{lcccc}
\hline & \multicolumn{3}{c}{ Guía Informativa de Alta } \\
\cline { 2 - 5 } Características & \multicolumn{2}{c}{ Aplica } & \multicolumn{2}{c}{ No Aplica } \\
\cline { 2 - 5 } & $\begin{array}{c}\text { Promedio } \pm \text { DS } \\
\text { (Mín. - Máx.) }\end{array}$ & $\begin{array}{c}\text { Promedio } \pm \text { DS } \\
\text { (Mín. - Máx.) }\end{array}$ \\
\hline Edad & $16,8 \pm 1,4$ & $(13-19)$ & $16,6 \pm 1,2$ & $(14-18)$ \\
Nivel educativo. & $\mathbf{N}$ & $\%$ & $\mathbf{N}$ & $\%$ \\
\hline Primaria & 4 & $13,3 \%$ & 3 & $10,0 \%$ \\
\hline Secundaria & 22 & $73,3 \%$ & 26 & $86,7 \%$ \\
\hline Superior Técnico & 0 & $.0 \%$ & 0 & $.0 \%$ \\
\hline Superior Universitario & 0 & $.0 \%$ & 1 & $3,3 \%$ \\
\hline Sin Instrucción & 4 & $13,3 \%$ & 0 & $.0 \%$ \\
\hline Estado civil. & & & & \\
\hline Soltera & 6 & $20,0 \%$ & 11 & $36,7 \%$ \\
\hline Casada & 1 & $3,3 \%$ & 0 & $.0 \%$ \\
\hline Conviviente & 23 & $76,7 \%$ & 19 & $63,3 \%$ \\
\hline \multicolumn{1}{c}{ Total } & $\mathbf{3 0}$ & $\mathbf{1 0 0 , 0} \%$ & $\mathbf{3 0}$ & $\mathbf{1 0 0 , 0} \%$ \\
\hline
\end{tabular}

Fuente: Instrumento 2 de la Guía Informativa de Alta. 
Tabla 4. Comparativo de Características obstétricas de las puérperas mediatas atendidas en el servicio de adolescencia del INMP- 2012.

\begin{tabular}{|c|c|c|c|c|}
\hline \multirow{3}{*}{$\begin{array}{c}\text { Características } \\
\text { obstétricas. }\end{array}$} & \multicolumn{4}{|c|}{ Guía Informativa de Alta } \\
\hline & \multicolumn{2}{|c|}{ Aplica } & \multicolumn{2}{|c|}{ No Aplica } \\
\hline & \multicolumn{2}{|c|}{$\begin{array}{l}\text { Promedio } \pm \text { DS } \\
\text { (Mín. - Máx.) }\end{array}$} & \multicolumn{2}{|c|}{$\begin{array}{l}\text { Promedio } \pm \text { DS } \\
\text { (Mín. - Máx.) }\end{array}$} \\
\hline Estancia Hospitalaria. & \multicolumn{2}{|c|}{$3,0 \pm 1,5(1-7)$} & \multicolumn{2}{|c|}{$3,9 \pm 1,4(2-7)$} \\
\hline Tipo de parto. & $\mathbf{N}$ & $\%$ & $\mathbf{N}$ & $\%$ \\
\hline Vaginal & 22 & $73,3 \%$ & 16 & $53,3 \%$ \\
\hline Cesárea & 8 & $26,7 \%$ & 14 & $46,7 \%$ \\
\hline Total & 30 & $100,0 \%$ & 30 & $100,0 \%$ \\
\hline
\end{tabular}

Fuente: Instrumento 2 de la Guía Informativa de Alta.

16,72 $\pm 1,318$ años con una edad mínima de 13 años y una máxima de 19

El $80 \%$ son de educación secundaria, el $11,7 \%$ tuvo nivel educativo primario y el $6,7 \%$ no tuvo ningún tipo de instrucción .Según el estado civil: $28,3 \%$ fueron solteras y $70 \%$ fueron convivientes (Tabla 2 y Figura 1).

Respecto a las características socio demográficas entre el grupo experimental y el grupo control son similares en edad promedio $(p=0,57)\left({ }^{1}\right)$, en nivel educativo $(p=0,14)^{2}$, en estado civil $(p=0,24)\left({ }^{2}\right)$. Es decir, la edad promedio en el grupo experimental $16,8 \pm 1,4$ es similar que el grupo control 16,6 $\pm 1,2$; los porcentajes de nivel educativo de primaria en el grupo experimental $(13,3 \%)$ es similar al del grupo control (10\%), nivel educativo de secundaria en el grupo experimental $(73,3 \%)$ es similar al del grupo control $(86,7 \%)$ y el porcentaje de estado civil de Soltera en el grupo experimental $(20 \%)$ es similar al del grupo control $(36,7 \%)$ y el porcentaje de estado civil de Conviviente en el grupo experimental $(76,7 \%)$ es similar al del grupo control $(63,3 \%)$ (Tabla 3).

Respecto a las características obstétricas entre el grupo experimental y el grupo control son similares en la estancia hospitalaria promedio $(p=0,34)\left({ }^{1}\right)$ y tipo de parto $(p=0,11)^{2}$. Es decir, la estancia hospitalaria promedio en el grupo experimental $3,0 \pm 1,5$ es similar que el grupo control 3,9 $\pm 1,4$; el porcentaje de tipo de parto vaginal en el grupo experimental $(73,3 \%)$ es similar al del grupo control $(53,3 \%)$ y el porcentaje de tipo de parto cesárea en el grupo experimental $(26,7 \%)$ es similar al del grupo control $(46,7 \%)$ (Tabla 4).

Los resultados de las tabla 3 y 4 permiten definir que las muestras evaluadas en el grupo experimental y del grupo control son similares en edad, nivel educativo, estado civil, tipo de parto y estancia hospitalaria, esto permite establecer que las muestras son similares en estas variables y que garantiza que se pueda interpretar los resultados de nivel de cumplimiento de las indicaciones de cuidado sin la posible duda de que pueda intervenir alguna de estas variables y sesgar las conclusiones finales.

Como se aprecia en la tabla 5 existen evidencias estadísticas con un nivel de confianza del $95 \%$ para afirmar que la calificación del nivel de cumplimiento en los cuidado puerperales y la aplicación o no de la guía de orientación se relacionan $(p<0,001)$; es decir, existe un efecto positivo de la implementación de la guía de informativa de alta en el cumplimiento de las indicaciones y cuidados puerperales y cuidados del recién nacido, en las pacientes adolescentes atendidas en el Servicio de Adolescencia del INMP.

Tabla 5. Nivel de Cumplimiento de las indicaciones y cuidados puerperales y del recién nacido de las adolescentes atendidas en el servicio de adolescencia del INMP- 2012.

\begin{tabular}{|c|c|c|c|c|c|c|c|c|}
\hline \multirow{3}{*}{\multicolumn{2}{|c|}{$\begin{array}{l}\text { Nivel de cumplimiento de las indicaciones } \\
\text { y cuidados en puérperas adolescentes y } \\
\text { cuidados del recién nacido. }\end{array}$}} & \multicolumn{6}{|c|}{ Guía Informativa de Alta } & \multirow{3}{*}{$p\left(^{*}\right)$} \\
\hline & & \multicolumn{2}{|c|}{ Aplica } & \multicolumn{2}{|c|}{ No Aplica } & \multicolumn{2}{|c|}{ Total } & \\
\hline & & $\mathbf{N}$ & $\%$ & $\mathbf{N}$ & $\%$ & $\mathbf{N}$ & $\%$ & \\
\hline \multirow{5}{*}{$\begin{array}{l}\text { Nivel de cumplimiento } \\
\text { de las indicaciones y } \\
\text { cuidados en puérperas } \\
\text { adolescentes. }\end{array}$} & Muy bajo (0-7) & 0 &, $0 \%$ & 0 & $.0 \%$ & 0 & $.0 \%$ & \multirow{5}{*}{$<0,001$} \\
\hline & Bajo (8-15) & 2 & $6,7 \%$ & 6 & $20,0 \%$ & 8 & $13,3 \%$ & \\
\hline & Regular (16-23) & 1 & $3,3 \%$ & 15 & $50,0 \%$ & 16 & $26,7 \%$ & \\
\hline & Bueno (24-30) & 8 & $26,7 \%$ & 7 & $23,3 \%$ & 15 & $25,0 \%$ & \\
\hline & Muy bueno (31-38) & 19 & $63,3 \%$ & 2 & $6,7 \%$ & 21 & $35,0 \%$ & \\
\hline \multirow{5}{*}{$\begin{array}{l}\text { Nivel de cumplimiento } \\
\text { de indicaciones y } \\
\text { cuidados al recién } \\
\text { nacido. }\end{array}$} & Muy bajo (0-4) & 0 & $.0 \%$ & 2 & $6,7 \%$ & 2 & $3,3 \%$ & \multirow{5}{*}{$<0,001$} \\
\hline & Bajo (5-9) & 0 & $.0 \%$ & 0 & $.0 \%$ & 0 & $.0 \%$ & \\
\hline & Regular (10-14) & 1 & $3,3 \%$ & 14 & $46,7 \%$ & 15 & $25,0 \%$ & \\
\hline & Bueno (15-19) & 9 & $30,0 \%$ & 9 & $30,0 \%$ & 18 & $30,0 \%$ & \\
\hline & Muy bueno (20-24) & 20 & $66,7 \%$ & 5 & $16,7 \%$ & 25 & $41,7 \%$ & \\
\hline \multicolumn{2}{|c|}{ Total } & 30 & $100,0 \%$ & 30 & $100,0 \%$ & 60 & $100,0 \%$ & \\
\hline
\end{tabular}

Fuente: Instrumento 2 de la Guía Informativa de Alta.

Prueba Chi-cuadrado ( $\left.{ }^{*}\right)$

\footnotetext{
( )Prueba T-student

( )Prueba Chi-cuadrado
} 
Cabe mencionar que en la evaluación del nivel de cumplimiento de las pacientes con respecto a los cuidados puerperales, en el grupo que se aplicó la guía de orientación se alcanzó el $63,3 \%$ de cumplimiento calificado como "muy bueno", comparado con el 6,7\% del grupo que no se aplicó la guía de orientación. Para el nivel de cumplimiento sobre los cuidados del recién nacido, en el grupo experimental se alcanzó el $66,7 \%$ de cumplimiento calificado como "muy bueno", comparado con el $16,7 \%$ del grupo control.

\section{DISCUSIÓN}

Según el estudio realizado por Sanabria ${ }^{13}$ se obtuvo como resultados que el promedio de edad materna fue de 24 años (rango 14-41). Similares características fueron encontrados por Amorin en cuanto al perfil personal y socio-demográfico de las pacientes en su estudio, pues las edades estuvieron comprendidas entre los 14 a 19 años, y el 59\% estaban en el rango de 15 a 18 años, con respecto al nivel educativo el $54 \%$ tenía educación primaria incompleta, el $50 \%$ no ejerce ningún tipo de trabajo remunerado, con sus actividades restringidas al ámbito doméstico: $35 \%$ realizan un trabajo remunerado fuera del hogar y sólo el $14 \%$ (tres) de las mujeres reportaron ser estudiantes. En la presente investigación la edad promedio de las pacientes fue de 16,72 $\pm 1,318$ años, teniendo el $80 \%$ de ellas educación secundaria y el $11,7 \%$ nivel primaria; el estado civil de la mayoría fue conviviente $(70 \%)$ y solo el $28,3 \%$ fueron solteras.

Sanabria y col $^{13}$ reportó que el número de controles prenatales promedio fue 5 ; el $22,8 \%$ tuvo menos de 4 consultas; el promedio de días de internación fue 2; el $58 \%$ de los partos fue por vía vaginal y el $42 \%$ por cesárea. En nuestro estudio los días de permanencia fueron $3,0 \pm 1,5$ y 3,9 $\pm 1,4$ respectivamente; asimismo, con respecto al tipo de parto: el porcentaje de tipo de parto vaginal en el grupo experimental $(73,3 \%)$ es similar al del grupo control $(53,3 \%)$ y el porcentaje de tipo de parto cesárea en el grupo experimental $(26,7 \%)$ es similar al del grupo control.

Calderón y col ${ }^{22}$ en el año 2008 obtuvo como resultados que durante el cuidado prenatal sólo el $39 \%$ de las madres recibió consejos sobre cómo cuidar el pezón; el $74 \%$ de las madres dió pecho a su hijo en la $1^{\text {a }}$ hora y el $70 \%$ recibió instrucciones sobre los beneficios de la lactancia materna (LM). El 83\% de las madres le dio a su hijo pecho materno exclusivo. Sin embargo, al $57,6 \%$ de las madres no se le orientó sobre dónde acudir en caso de dificultades con la LM. Se observó en relación a los signos de agarre: $44,2 \%$ de los RN no tocaba el pecho materno con el mentón, $31,4 \%$ no tenían la boca bien abierta: $40,9 \%$ no tenía el labio evertido y $45,7 \%$ se veía menos areola por arriba de la boca, de este grupo el $50 \%$ de las pacientes tuvo una guía informativa previa a la aplicación de la encuesta mientras que el otro grupo no tuvo ninguna orientación con la guía. Por lo que en el grupo al que se le brindo la información con la guía antes de la intervención predominó un nivel medio $(52,3 \%)$ de conocimiento con proporciones mayores a $46,5 \%$ en cada dimensión y puntaje mínimo en cada rango, con una media global de $24,5 \pm 10,4$; después de la intervención predominó el nivel alto en el $100 \%$ de la población, con una media global de $94,5 \pm 1,2$, concluyendo que: la intervención personalizada influyó significativamente en el nivel de conocimiento de las adolescentes sobre las prácticas de auto cuidado en el puerperio. En el estudio se encontró similares resultados en adolescentes con respecto a la lactancia materna donde muchas adolescentes del primer grupo (grupo experimental) utilizaron la técnica sentada con apoyo $(46,7 \%)$ y en el segundo grupo (grupo comparativo) la mayoría utilizo todo tipo de técnicas. Respecto al uso de la técnica de amamantamiento que le enseñó el INMP, el $100 \%$ de las pacientes del grupo al que se aplicó la guía informativa lo realizó, a diferencia del $80 \%$ que se obtuvo del grupo al cual no se aplicó la guía. En cuanto al lavado de manos antes de dar de lactar al bebé, dentro del grupo al cual se aplicó la guía, un $70 \%$ lo realizó; mientras que solo un $43,3 \%$ del grupo al cual no se aplicó la guía, realizó esta acción, evaluándose que el nivel de cumplimiento de las pacientes con respecto a los cuidados puerperales, en el grupo que se aplicó la guía de orientación se alcanzó el $63,3 \%$ de cumplimiento calificado como "muy bueno", comparado con el 6,7\% del grupo que no se aplicó la guía de orientación. Para el nivel de cumplimiento sobre los cuidados del recién nacido, en el grupo experimental se alcanzó el 66,7\% de cumplimiento calificado como "muy bueno", comparado con el $16,7 \%$ del grupo control.

En conclusión, se puede apreciar que el nivel de cumplimiento de la guía de orientación sobre el cuidado puerperal y del recién nacido alcanzó efecto positivo relevante.

\section{REFERENCIAS BIBLIOGRÁFICAS}

1. Organización Mundial de la Salud [en línea].Suiza: Ginebra .2011. [Accesado 20 Mayo 2012]. Salud y desarrollo del niño y del adolescente. [aproximadamente 1 pantallas]. Disponible en: http://www.who.int/child_adolescent_health/topics/ prevention_care/adoles ent/es/

2. Donoso E. Mortalidad materna, Chile 2005 (Editorial). Rev Chil Obstet Ginecol 2008; 73(3): 143-144. Disponible en:

http://www.scielo.cl/scielo.php?pid=S071775262009000400 001\&script=sci_arttext.

3. Plan estratégico nacional para la reducción de la mortalidad materna y perinatal 2009-2015 Dirección General de Salud de las Personas. Estrategia Sanitaria Nacional de Salud Sexual y Reproductiva - Lima: Ministerio de Salud; 2009. 56 p. Reproductiva - Lima: Ministerio de Salud; 2009.

4. Encuesta Demográfica y de Salud Familiar [en línea]. Perú: Lima. 2009 [Accesado 19 de Mayo 2012]. Embarazo en adolescente. [aproximadamente 3 pantallas]. Disponible en : http://desa.inei.gob.pe/endes/

5. Organización Mundial de la Salud [en línea]. Suiza: Ginebra .2009 [Accesado 19 de Mayo 2012]. Embarazo en adolescente: un problema culturalmente complejo. 
[aproximadamente 1 pantallas]. Disponible en: http://www. who.int/bulletin/volumes/87/6/09-020609/es/

6. Organización Mundial de la Salud [en línea].Suiza: Ginebra 2011 [Accesado 12 Mayo 2011]. 10 Datos sobre salud de los adolescentes. Disponible en : http://www.who.int/features/ factfiles/adolescent health/es/index.html

7. Orientaciones para la atención integral de salud del adolescente en el primer nivel de atención Dirección General de Salud de las Personas. Dirección de Atención Integral. Etapa de Vida Adolescente -- Lima: Ministerio de Salud; 2007. 86 p. Documento técnico RM N¹077-2006/MINSA.

8. Martínez Pérez A. Modificación de los conocimientos de las madres adolescentes sobre los cuidados básicos al recién nacido después de la aplicación de un programa educativo [tesis de Grado]. Colombia: Pontificia Universidad Javeriana, Facultad de Enfermería; 2009.

9. Zalles J. Protocolos de atención materna y neonatal culturalmente adecuados. Serie de documentos TécnicoNormativos "Normas y Protocolos". Bolivia 2006.

10. Betancourt, H. Guías de atención de los principales problemas de salud de adolescentes. Ministerio de salud pública y asistencia social. dirección de regulación. El Salvador 2003.

11. Romanzini A., Marcielo de Jesús A., Carvalho E., Menis V., Damiano V., Gomes J. Directrices de enfermería a los pacientes sobre el autocuidado y las señales y síntomas de una infección en la herida para la post alta hospitalaria de una cirugía cardíaca reconstructora. Reme - Rev. Min. Enferm.; 14(2): 239-243, abr. /jun., 2010.

12. Céspedes LM., Sáenz X. "Plan de alta para personas con enfermedad pulmonar obstructiva crónica". Aquichan, octubre, año/vol. 6, numero 001. Universidad de La Sabana Chía, Colombia. PP.104- 106. Disponible en: http://redalyc. uaemex.mx/pdf/741/74160111.pdf.

13. Sanabria M., Coronel J., Salinas C., Sartori J. "Perfil de la lactancia materna en cuatro servicios de referencia neonatal". Universidad Nacional de la Asunción. Arch Pediatr Urug 2005; 76(4): 340-345

14. Amorim da Silva L., Spanó AM., Azevedo FG, Stefanello J. "Los significados asignados por la maternidad a las madres adolescentes: autocuidado y el cuidado del bebe". Hospital de Maternidad. Brasil. Texto Contexto Enferm, Florianópolis, 2009 Jan-Mar; 18(1): 48-56.

15. Cardozo S. Sandra, Bernal R. María. Adolescentes en el puerperio y sus prácticas de cuidado. Av. Enferm. XXVII (2): 82-91, 2009.
16. Molina C.; Viriam LD. "Necesidades educativas de las madres adolescentes acerca de la etapa de posparto". Rev. Enfermería Actual en Costa Rica [en línea].2010, No.18 [citado (fecha)]. Disponible: World Wide Web: <http://www. revenf.ucr.ac.cr/necesidadeseducativas.pdf $>$ ISSN 14094568.

17. Unicef. Estado Mundial de la Infancia (EMI) 2009. Salud materna y neonatal. Presentación en proyecto Febrero 2009. Disponible en: http://www.unicef.org/devpro/files/ SOWC_2011_Main_Report_SP_02092011.pdf

18. Organización Mundial de la Salud [en línea]. Suiza: Ginebra .2011 [accesado 06 Ago. 2011]. Salud de los adolescentes. Disponible en : http://www.who.int/topics/adolescent health/ es/

19. Valverde R. "Guía de Atención Integral a las Mujeres Niños y Niñas en el periodo prenatal, parto y posparto". Costa Rica2008 Disponible en: http://www.mamasol.com/uploaded/ content/category/1090515659.pdf.

20. Orem D. Factores Que Condicionan A las madres adolescentes a tener un déficit de autocuidado en el recién nacido. Washington 1980. Ciencia y Cuidado Volumen 4N4. ISSN 37949083.

21. Ramírez MC, Díaz de Correa Y, Luna MA. El profesional de enfermería en la vigilancia epidemiológica de las infecciones intrahospitalarias. Guías ACOFAEN. Biblioteca Las casas, 2005; 1. Disponible en http://www.index-f.com/lascasas/ documentos/lc0019.php.

22. Calderón $Y$, Requejo D. Influencias de una intervención educativa en el nivel de conocimiento sobre prácticas de autocuidado durante el puerperio en adolescentes. Rev Enferm Herediana 2008;1 (1): 11

23. Fondo de las Naciones Unidas para la Infancia [en línea]. EE.UU: NY. 2011 [accesado 06 Ago 2011]. La adolescencia: Una época de oportunidades. Disponible:http://www.unicef.org/ devpro/files/SOWC_2011_Main_Report_SP_02092011.pdf.

24. Medrano M y col. Niños: Guía de atención para el menor de 5 años. Lima-Perú 2002.

25. Andagana K. Guía de atención de enfermería quirúrgica en pacientes craneotomizados en el hospital de Ambato. Tesis para obtener el título de magister en enfermería quirúrgica. Ambato- Ecuador.2011.

26. Basantes V. Guía de atención de enfermería en el proceso quirúrgico en el hospital provincial general docente de Riobamba. Tesis de grado para obtención de título en enfermería quirúrgica. Ambato- Ecuador. 2010. 